Patricky Santos Silva ${ }^{1}$ Brena da Costa Campos Range ${ }^{1}$ Selma Rodrigues de Castilho ${ }^{1}$

\title{
AVALIAÇÃO DA PROPAGANDA DE MEDICAMENTOS ISENTOS DE PRESCRIÇÃO EM FARMÁCIAS COMUNITÁRIAS DO MUNICÍPIO DE NITERÓI (RJ, BRASIL)
}

Evaluation of over-the-counter medicines' advertising in community pharmacies in the city of Niterói (RJ, Brazil)

${ }^{1}$ Universidade Federal Fluminense. Niterói/RJ, Brasil.

Correspondência: Selma Rodrigues de Castilho.E-mail: selmarc@id.uff.br.

Recebido: 12/12/2016. Revisado: 07/04/2017. Aprovado: 04/05/2017. 


\section{RESUMO}

O presente trabalho trata-se de estudo transversal para coleta e análise do cumprimento da legislação sanitária em peças de propaganda de medicamentos de venda livre, destinadas aos consumidores, veiculadas em farmácias e drogarias privadas no município de Niterói. Das 826 peças publicitárias de medicamentos coletadas em 80 farmácias, 379 eram inéditas; aproximadamente $99,5 \%$ das peças apresentaram algum tipo de irregularidade frente à RDC n. 96/2008, com média de 5,1 infrações por peça analisada, sendo 16 o número máximo de infrações observadas em uma única peça. Os resultados apontam para o risco que o atual panorama da propaganda de medicamentos no país oferece à população, diariamente exposta a essa fonte de (des)informação, e para a necessidade de uma fiscalização mais efetiva da propaganda de medicamentos direcionada a público leigo.

\section{Palavras-Chave}

Legislação Sanitária; Medicamentos; Propaganda.

\section{ABSTRACT}

Cross-sectional study for collection and analysis of the compliance with health legislation of OTC drug advertising, for consumers, available in pharmacies and private drugstores in the city of Niterói (Rio de Janeiro, Brazil). Of the 826 medicines' advertisements collected from 80 pharmacies, 379 were new. Approximately $99.5 \%$ of these pieces had some kind of irregularity according to the Brazilian Health Regulatory Agency Resolution n. 96/2008, averaging 5.1 offenses by piece analyzed, being 16 the maximum number of violations observed in a single piece. The results point to the risk imposed by the current landscape of drug advertising in the country to the population, daily exposed to this source of misinformation, and to the need for a more effective supervision of drug advertising that is directed to the lay public.

\section{Keywords}

Advertising; Health Legislation; Medicines. 


\section{Introdução}

Todo indivíduo que vive em uma sociedade ocidental capitalista terá acesso a algum tipo de propaganda durante sua vida, incluindo algum tipo de propaganda de medicamentos ${ }^{1}$. As propagandas de medicamentos, em sua maioria, visam não apenas à apresentação do produto como a uma opção para o tratamento de determinada enfermidade; hoje, mais do que nunca, visam à exploração de desejos e sentimentos abstratos, advindos do simbolismo embutido nessas tecnologias, estabelecido principalmente no conceito de cura instantânea, mágica ${ }^{2}$. Nesse contexto, a indústria farmacêutica tem investido cifras cada vez maiores em propaganda e publicidade para a valorização e divulgação de seus produtos ${ }^{3}$.

Além disso, estudos têm demonstrado a influência da propaganda e publicidade de medicamentos na vida das pessoas ${ }^{4},{ }_{5}$. Entretanto, essa ferramenta tem sido uma prática produtora de risco. Estudos realizados sobre a qualidade das peças publicitárias de medicamentos - em diferentes meios de comunicação e dirigidas a diferentes populações-alvo - têm demonstrado que a grande maioria das propagandas está em desacordo com a legislação vigente $e^{6,7,8}$.

A propaganda de medicamentos direcionada aos consumidores é tratada de forma diferenciada no mundo. Enquanto no Brasil, na Coreia do Sul e nos países da União Europeia, por exemplo, a veiculação de propagandas ao consumidor é restrita

\footnotetext{
${ }^{1}$ DEL MONTE, Michele Helena Lopes. A influência da publicidade televisiva no consumo da população de classe C: os anunciantes do varejo popular. 2011. Dissertação (Mestrado) - Escola de Comunicações e Artes, Universidade de São Paulo. São Paulo, 2011.

${ }^{2}$ LĖFREVE, Fernando. A função simbólica dos medicamentos. Revista de Saúde Pública, São Paulo, v. 17, n. 6, p. 500-503, 1983. Disponível em: <http://www.scielo.br/pdf/rsp/v17n6/07.pdf>. http://dx.doi. org/10.1590/S0034-89101983000600007.

3SINDICATO DA INDÚSTRIA DE PRODUTOS FARMACÊUTICOS (São Paulo). Disponível em: <http://www. sindusfarmacomunica.org.br/indicadores-economicos/>. Acesso em: 05 ago. 2013.

${ }^{4}$ PECHMANN, Cornelia; CATLIN, Jesse R.The effects of advertising and other marketing communications on health-related consumer behaviors.Current Opinion in Psychology USA, v.10, p. 44-49, 2016.https://doi. org/10.1016/j.copsyc.2015.12.008.

${ }^{5}$ NEVES, Aline Souza; LYRA, Divaldo Pereira; CERQUEIRA, Karine Santos; MARCELLINE, Paulo Sérgio; MARQUES, Tatiane Cristina; BARROS, José Augusto Cabral. Influência da propaganda na utilização de medicamentos em grupo de idosos atendidos em uma Unidade Básica de Saúde em Aracaju (SE, Brasil). Ciência \& Saúde Coletiva, Brasil, v. 15, n. 3, p. 3497-3505, 2010. Disponível em: <http://www.scielo.br/ pdf/csc/v15s3/v15s3a24.pdf>. http://dx.doi.org/10.1590/S1413-81232010000900024.

${ }^{6}$ AZEVEDO, Germana Santos; PEREIRA, Orcione Aparecida Vieira. Análise da publicidade das propagandas de medicamentos veiculadas em emissoras de televisão. Farmácia \& Ciência, Brasil, v. 1, p. 3-15, 2010. Disponível em: <https://www.unilestemg.br/farmaciaeciencia/volumes/artigo_1_F_C.pdf>.

${ }^{7}$ CARVALHO, Marselle Nobre; BARROS, José Augusto Cabral. Propagandas de medicamentos em revistas femininas. Saúde em Debate, Rio de Janeiro, v. 37, n. 96, p. 76-83, jan./mar. 2013. Disponível em: <http:// www.scielo.br/pdf/sdeb/v37n96/09.pdf>. http://dx.doi.org/10.1590/S0103-11042013000100009.

${ }^{8}$ ABDALLA, Marcela Campos Esqueff. Monitoramento da propaganda de medicamentos direcionada a profissionais de saúde do Município de Niterói-RJ. 2015. Dissertação (Mestrado) - Faculdade de Farmácia, Universidade Federal Fluminense, Niterói, 2015.
} 
apenas aos medicamentos isentos de prescrição (MIP $)^{9,10,11,12}$, nos Estados Unidos e na Nova Zelândia também é facultada a propaganda para público leigo de produtos sob prescrição médica ${ }^{13}$. Nos Estados Unidos, o Food and Drug Administration (FDA), divide em três categorias a publicidade de medicamentos direcionada ao público leigo: (a) os lembretes (que basicamente se destinam a lembrar o nome de um produto), (b) os anúncios de ajuda à busca da informação (trazem informação sobre uma doença ou condição, sem mencionar um tratamento em particular); e (c) os anúncios de produtos, que trazem informações sobre a segurança e eficácia de um produto específico. Apenas esta última categoria é regulada, sendo exigido equilíbrio adequado entre os riscos e benefícios apresentados pela peça publicitária ${ }^{13}$.

A Resolução da Diretoria Colegiada da Agência Nacional de Vigilância Sanitária (Anvisa) n. 96/2008 (RDC n. 96/2008) apresenta um capítulo específico sobre a regulação dos MIP, trazendo uma série de parâmetros a serem seguidos ${ }^{9}$. Farmácias comunitárias usualmente apresentam em seu interior peças publicitárias sobre MIP, sendo objetivo deste trabalho analisar a qualidade dessas peças publicitárias frente à RDC n. 96/2008.

\section{Fundamentação teórica}

No Brasil, a RDC n. 96/2008 é o atual mecanismo regulador da propaganda, publicidade, informação e outras práticas cujo objetivo seja divulgar ou promover comercialmente os medicamentos ${ }^{9}$. Vários autores evidenciaram a baixa qualidade de peças publicitárias veiculadas no Brasil tomando por base esse arcabouço legal ${ }^{6,7,8,14}$. O maior estudo sobre a situação da propaganda de medicamentos no país foi o realizado pela Anvisa em conjunto com instituições de ensino superior de todo o país, denominado Projeto de Monitoração de Propaganda ${ }^{14}$. Pôde-se observar a baixa

${ }^{9}$ BRASIL. Agência Nacional de Vigilância Sanitária - ANVISA. Resolução RDC n. 96, de 17 de dezembro de 2008. Dispõe sobre a propaganda, publicidade, informação e outras práticas cujo objetivo seja a divulgação ou promoção comercial de medicamentos. Disponível em: <http://www.anvisa.gov.br/propaganda/rdc/ rdc_96_2008_consolidada.pdf>. Acesso em: 09 maio 2017.

${ }^{10}$ FROSCH, Dominick L, GRANDE, David; TARN, Derjung M.; KRAVITZ, Richard L. A decade of controversy: balancing policy with evidence in the regulation of prescription drug advertising. American Journal of Public Health, v. 100, n. 1, p. 24-32, 2010. Disponível em: <https://www.ncbi.nlm.nih.gov/pmc/articles/ PMC2791253/pdf/24.pdf>.10.2105/AJPH.2008.153767.

${ }^{11}$ SUH, Hae Sun; LEE, Donghyun; KIM, Sam Yong; CHEE, Dong Hyun; KANG, Hye-Young. Direct-to-consumer advertising (DTCA) for prescription drugs: Consumers' attitudes and preferences concerning its regulation in South Korea. Health Policy, v. 101, p. 260-268, 2011. 10.1016/j.healthpol.2011.05.005.

${ }^{12}$ GEYER, Robert. The politics of EU health policy and the case of direct-to-consumer advertising for prescription drugs. British Journal of Politics and International Relations (BJPIR), v. 13, n. 4, p. 586-602, Nov. 2011.10.1111/j.1467-856X.2011.00460.x.

${ }^{13}$ GELLAD, Ziad F., LYLES, Kenneth W. Direct-to-consumer advertising of pharmaceuticals. The American Journal of Medicine, v. 120, p. 475-480, Jun. 2007.10.1016/j.amjmed.2006.09.0.

${ }^{14}$ BRASIL. Agência Nacional de Vigilância Sanitária - ANVISA. Monitoração de propaganda: relatório final do projeto de monitoração. Brasília, 2010. Disponível em: <http://portal.anvisa.gov.br/ documents/33864/285104/RelatorioGestaoGPROP_2010_baixa_11072011.pdf/1972dc29-b15f-48af854b-4403f8ae697d?version=1.0>. 
qualidade das peças publicitárias, cuja grande maioria não respeitava, em algum ponto, a legislação vigente. As infrações mais recorrentes nas peças publicitárias foram ausência de contraindicações, ausência do número de registro do produto junto à Anvisa e ausência de advertências. Essas irregularidades levam, entre outros riscos, ao uso irracional de medicamentos. Isso porque, com a ausência dessas informações, o indivíduo que utiliza a propaganda como fonte de conhecimento tende a adquirir e utilizar o medicamento com a falsa ideia de que o mesmo não apresenta riscos, podendo ainda não escolher o medicamento de venda livre mais adequado para seu estado de saúde. Todavia, ao longo das fases do projeto de monitoração, observou-se uma diminuição na incidência de determinadas infrações.

Nascimento $^{15}$ aponta que propagandas irregulares ocasionam risco, uma vez que são importantes fontes de informação para grande parcela da população. Sendo as principais estratégias das propagandas a supervalorização dos benefícios e a ocultação dos riscos. Sabendo-se que nenhum medicamento é inócuo, há de fato risco no uso irracional de medicamentos estimulados por propagandas, principalmente aquelas irregulares.

Ausência da data de impressão das peças publicitárias, dificuldade de visualização de informações das peças e ausência ou não conformidade do número de registro do produto junto à Anvisa estão entre as infrações mais cometidas pela propaganda monitorada. Essas irregularidades (a) podem levar à dificuldade de identificar o momento da infração e há quanto tempo a peça infringe a lei, (b) promovem uso irracional de medicamentos e (c) praticam fraude, colocando em risco a saúde da população atingida. A baixa qualidade de peças publicitárias de medicamentos também é evidenciada em outros países, seja na propaganda direcionada aos profissionais de saúde ${ }^{16}$, seja na propaganda direcionada a público leigo ${ }^{17}{ }^{18}$. Entre os problemas citados, encontram-se a veiculação de informação incompleta, a ausência da data de publicação da peça publicitária e o desequilíbrio entre as informações sobre os efeitos benéficos e os riscos dos produtos.

\footnotetext{
${ }^{15}$ NASCIMENTO, Álvaro César. Propaganda de medicamentos no Brasil: é possível regular? Ciência \& Saúde Coletiva, Brasil, v. 14, n. 3, p. 869-877, maio/jun. 2009. Disponível em: <http://www.scielo.br/pdf/csc/ v14n3/en_22.pdf>. http://dx.doi.org/10.1590/S1413-81232009000300022.

${ }^{16}$ KORENSTEIN, Deborah, KEYHANI, Salomeh, MENDELSON, Ali, ROSS, Joseph S. Adherence of Pharmaceutical Advertisements. Medical Journals to FDA Guidelines and Content for Safe Prescribing. PloS ONE, v. 6, n. 8 e23336, Aug. 2011. Disponível em: <http://journals.plos.org/plosone/article/file?id=10.1371/journal. pone.0023336\&type=printable>. https://doi.org/10.1371/journal.pone.0023336.

${ }^{17}$ AL-AGEEL, Sinaa Abdul Mohsen; AL-SABHAN, Jawza Fahad, SULTAN, NohaYahia. Analysis of written advertising material distributed through community pharmacies in Riyadh, Saudi Arabia. PharmPract, Granada, v.11, n. 3, p. 138-143, Jul./Sep. 2013. Disponível em: <https://www.ncbi.nlm.nih.gov/pmc/ articles/PMC3809137/pdf/pharmpract-11-138.pdf>.

${ }^{18}$ ADIBE, Maxwell Ogochukwu; IGBOELI, NnekaUchenna; UBAKA, Chukwuemeka Michael; UDEOGARANYA, Patrick Obinna; ONWUDIWE, NkejahPrecious; ITA, OboOkokon. Evaluation of information contained in drug advertisement and promotion materials in Nigeria. Tropical Journal of Pharmaceutical Research, v. 14, n. 3, p. 539-544, 2015. Disponivel em: <https://www.ajol.info/index.php/tjpr/article/ download/115797/105359>. http://dx.doi.org/10.4314/tjpr.v14i3.25.
} 
Farmácias comunitárias frequentemente dispõem, em seu interior, de peças publicitárias destinadas ao público leigo. No entanto, não foram localizados estudos envolvendo a análise das propagandas de medicamentos presentes no interior desses estabelecimentos no Brasil. O estudo que mais se aproxima desse tipo de análise foi o realizado por Galato et al. ${ }^{19}$ envolvendo a análise dos encartes distribuídos em farmácias e drogarias do município de Tubarão, Santa Catarina, frente à RDC n. $102 / 2000^{20}$ e à RDC n. 96/2008. Os autores evidenciaram que a maioria das peças analisadas continha algum tipo de irregularidade frente à legislação, sendo as mais frequentes: não conformidade ou ausência do número de registro do produto no Ministério da Saúde (77,9\%) e ausência das contraindicações (54,3\%).

Adibe et al. ${ }^{21}$ estudaram a legibilidade e a qualidade de panfletos e material promocional coletados em farmácias e drogarias na Nigéria. Os autores observaram problemas como fornecimento de informação incompleta e inadequação do material em termos de legibilidade, além de exageros nos benefícios e minimização de riscos associados aos produtos anunciados.

\section{Metodologia}

Estudo transversal para coleta e análise do cumprimento da legislação sanitária em peças de propaganda de medicamentos de venda livre, destinadas aos consumidores, veiculadas em farmácias e drogarias privadas no município de Niterói. O período de coleta ocorreu entre os meses de janeiro e julho de 2016, e foram consideradas peças físicas disponibilizadas ao público e fotos de peças expostas nos estabelecimentos.

Os estabelecimentos foram identificados a partir da listagem fornecida pelo site Telelistas.net ${ }^{22}$, usando-se o descritor "drogaria" e os refinamentos por estado (RJ) e cidade (Niterói). Do total de 437 drogarias identificadas, 225 foram excluídas por serem estabelecimentos repetidos, estabelecimentos não pertencentes ao município do estudo, estabelecimentos que trabalhavam apenas com manipulação e estabelecimentos não mais existentes.

\footnotetext{
${ }^{19}$ GALATO, Dayani; PEREIRA, Greicy Borges; VALGAS, Cleidson. Análise de Informes Publicitários Distribuídos em Farmácias e Drogarias. Revista de Saúde Pública, São Paulo, v. 45, n. 1, p. 212-215, fev. 2011. Disponível em: <http://www.scielo.br/pdf/rsp/v45n1/en_1466.pdf>.http://dx.doi.org/10.1590/S003489102011000100024

${ }^{20}$ BRASIL. Agência Nacional de Vigilância Sanitária - ANVISA. Resolução RDC n. 102, de 30 de novembro de 2000. Dispõe sobre propagandas, mensagens publicitárias e promocionais e outras práticas cujo objeto seja a divulgação, promoção e/ou comercialização de medicamentos, de produção nacional ou importados, quaisquer que sejam suas formas e meios de veiculação incluindo as transmitidas no decorrer da programação normal das emissoras de rádio e televisão. Disponível em: <http://portal.anvisa.gov.br/ legislacao\#/visualizar/26616>. Acesso em: 02 fev. 2018.

${ }^{21}$ ADIBE, Maxwell Ogochukwu; IGBOELI, NnekaUchenna; UBAKA, Chukwuemeka Michael; UDEOGARANYA, Patrick Obinna; ONWUDIWE, NkejahPrecious; ITA, OboOkokon. op. cit.

${ }^{22}$ TELELISTAS.NET. Disponível em: <http://www.telelistas.net/>. Acesso em: 01 out. 2014.
} 
A cada estabelecimento, foi atribuída uma numeração; um sorteio único foi realizado para a seleção aleatória de 80 farmácias para a coleta de peças publicitárias e entrevistas. Não houve novo sorteio devido à seleção de farmácias da mesma rede ou do mesmo bairro nem pelo fato de alguma região não ter sido contemplada no estudo. A aleatoriedade do sorteio dos estabelecimentos foi obtida por meio do programa RandomNumberGenerator ${ }^{23}$.

Uma vez sorteada, as farmácias ou drogarias foram visitadas para coleta e/ ou registro fotográfico das peças publicitárias, sendo considerados tanto materiais provenientes de laboratórios, distribuidores e do próprio estabelecimento quanto fotos de fachadas, vitrines ou outras estratégias de apresentação de propaganda nos estabelecimentos. As fotos não identificaram o estabelecimento ou qualquer um de seus funcionários. Também foram coletados encartes de preços, panfletos e fôlderes das próprias farmácias.

A avaliação da adequação do material coletado com a RDC n. 96/2008 foi realizada com base no Manual de Monitoramento de Propaganda de Produtos Sujeitos à Vigilância Sanitária, disponibilizado pela Anvisa, por meio de seu cheklist ${ }^{24}$.

Para análise da compatibilidade das informações prestadas na peça com aquelas registradas na Anvisa, tomou-se por base o bulário eletrônico da Anvisa. Como a última atualização do bulário eletrônico da Anvisa data do ano de 2013, para medicamentos lançados após essa data a informação da peça foi comparada àquelas disponíveis na base de dados Drugdex ${ }^{25}$, da Micromedex.

Quanto à disponibilidade das referências bibliográficas no Serviço de Atendimento ao Consumidor (SAC) e no serviço de atendimento aos profissionais prescritores e dispensadores de medicamentos, consideraram-se os sites dos próprios fabricantes dos medicamentos em análise ou, em caso de existência, o site do medicamento em questão.

Os dados foram reunidos em um banco de dados e analisados a partir do programa Microsoft Excel 2007. Foram empregadas as ferramentas da estatística descritiva, sendo calculadas a frequência de irregularidades das propagandas e a distribuição dos grupos farmacológicos presentes nas propagandas, entre outros aspectos.

\footnotetext{
${ }^{23}$ RANDOM NUMBER GENARATOR Disponível em: <http://randomnumbergenerator.intemodino.com/pt/ gerador-de-numeros-aleatorios.html>. Acesso em: 08 out. 2015.

${ }^{24}$ BRASIL. Agência Nacional de Vigilância Sanitária - ANVISA. Gerência de Monitoramento e Fiscalização de Propaganda, Publicidade, Promoção e Informação de Produtos Sujeitos à Vigilância Sanitária. Manual: monitoramento de propaganda de produtos sujeitos à vigilância sanitária. Brasília-DF: Ministério da Saúde, 2005. (Série A. Normas e Manuais Técnicos). Disponível em: <http://www.anvisa.gov.br/propaganda/ manual_propaganda.pdf>.

${ }^{25}$ DRUGDEX. MicromedexDatabase. Disponível em: <http://www.library.auckland.ac.nz/databases/?FROM_ $\mathrm{REF}=\mathrm{BN}>$. Acesso em: 18 jul. 2016.
} 


\section{Resultados}

Foi coletado um total de 826 peças publicitárias de medicamentos, das quais 379 não se repetiam. Aproximadamente 99,5\% das inserções apresentaram algum tipo de irregularidade frente à RDC n. 96/2008, com média de 5,1 infrações e moda de cinco infrações por peça analisada, sendo 16 o número máximo de infrações observadas em uma única peça. Aproximadamente $75 \%$ das peças publicitárias tinham entre uma e seis infrações.

As 379 peças inéditas analisadas divulgavam 180 marcas diferentes de medicamentos. Dois analgésicos foram responsáveis por um total de 35 peças publicitárias, enquanto dois antiácidos responderam por 32 peças publicitárias e um expectorante, por 17 peças.

A classe terapêutica presente no maior número de peças publicitárias coletadas foi a dos fármacos com ação no trato gastrointestinal, representando $24,6 \%$ das propagandas coletadas (Gráfico 1). Em seguida, vieram os medicamentos com ação analgésica e/ou antitérmica, presentes em $24,5 \%$ do total de peças, e os polivitamínicos $(22,8 \%)$.

A peça publicitária com o maior número de infrações (16) não informava posologia, modo de usar e reações adversas, entre outras informações, todas de suma importância para o usuário. Além disso, não apresentava o nome da substância ativa, registro na Anvisa, data de impressão da peça, a advertência "Se persistirem os sintomas, o médico deverá ser consultado" e advertência relacionada à substância ativa. Não obstante, apresentava imagem de pessoa leiga em Farmácia ou Medicina, reconhecidamente famosa, sugerindo que utiliza o medicamento e relacionando-o ao alívio de excessos gastronômicos.

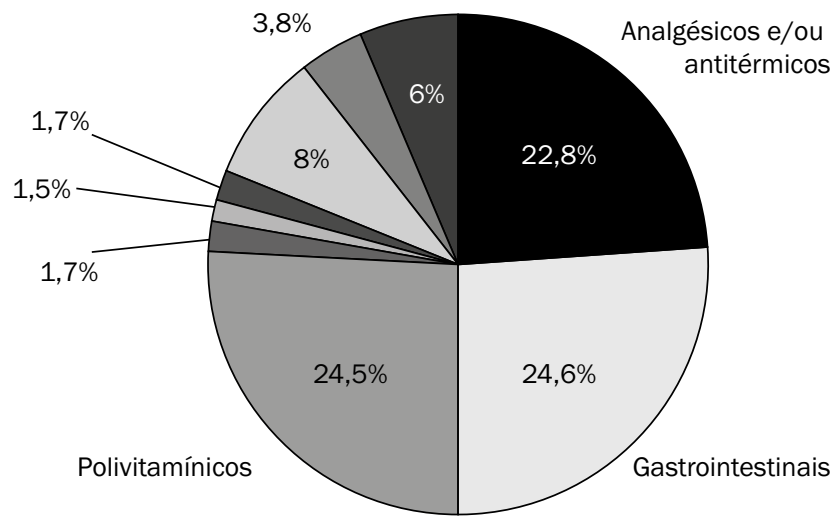

Gráfico 1. Classes terapêuticas envolvidas nas peças analisadas 
As tabelas 1 e 2 apresentam, respectivamente, os pontos da legislação mais e menos infringidos.

Tabela 1. Pontos da legislação mais infringidos pelas peças publicitárias

\begin{tabular}{|c|c|}
\hline Artigos (incisos) e suas determinações & $\begin{array}{c}\text { Porcentagem de } \\
\text { peças que infringiram }\end{array}$ \\
\hline Art. 22 (VI): data de impressão das peças publicitárias & $62 \%$ \\
\hline $\begin{array}{l}\text { Art. } 6^{\circ} \text { : permitem a sua imediata visualização, guardando entre si as } \\
\text { devidas proporções de distância, indispensáveis à legibilidade e destaque? }\end{array}$ & $57,5 \%$ \\
\hline $\begin{array}{l}\text { Art. } 22 \text { (VII): a advertência: "SE PERSISTIREM OS SINTOMAS, O MÉDICO } \\
\text { DEVERÁ SER CONSULTADO"? (observar o art. 6). }\end{array}$ & $45,4 \%$ \\
\hline Art. $22(\mathrm{~V})$ : as indicações & $44,3 \%$ \\
\hline $\begin{array}{l}\text { Art. 24: a advertência a que se refere o artigo } 23 \text { deve ser } \\
\text { contextualizada na peça publicitária, de maneira que seja pronunciada } \\
\text { pelo personagem principal, quando veiculada na televisão; proferida } \\
\text { pelo mesmo locutor, quando veiculada em rádio; e, quando impressa, } \\
\text { deve causar o mesmo impacto visual que as demais informações } \\
\text { presentes na peça publicitária, apresentando-se com, no mínimo, 35\% } \\
\text { do tamanho da maior fonte utilizada. }\end{array}$ & $35,4 \%$ \\
\hline
\end{tabular}

Fonte: Autoria própria.

Tabela 2. Pontos da legislação menos infringidos pelas propagandas analisadas e número de peças que infringiram.

\begin{tabular}{lc}
\hline Artigos (incisos) e suas determinações & $\begin{array}{c}\text { Número de peças que } \\
\text { infringiram }\end{array}$ \\
\hline $\begin{array}{l}\text { Art. } 26 \text { (II):sugerem que o medicamento é a única alternativa de } \\
\text { tratamento e/ou fazer crer que são supérfluos os hábitos de vida }\end{array}$ & 2 \\
saudáveis e/ou a consulta ao médico? & 1 \\
$\begin{array}{l}\text { Art. } 21 \text { (VI): apresentam de forma abusiva, enganosa ou assustadora } \\
\text { representações visuais das alterações do corpo humano causadas por } \\
\text { doenças ou lesões? }\end{array}$ & 2 \\
Art. $8^{\circ}$ (XI): usam expressões ou imagens que possam sugerir que a saúde \\
de uma pessoa poderá ser afetada por não usar o medicamento? \\
$\begin{array}{l}\text { Art. } 26 \text { (IV): usam linguagem direta ou indireta relacionando o uso de } \\
\text { medicamento a excessos etílicos ou gastronômicos? }\end{array}$ \\
\begin{tabular}{l} 
Art. $8^{\circ}$ (III): incluem imagens de pessoas fazendo uso do medicamento? \\
\hline
\end{tabular} \\
\hline
\end{tabular}

Fonte: Autoria própria.

\section{Discussão}

As peças estavam dispostas tanto no interior das drogarias como em suas fachadas, em gôndolas e nos caixas, assim como nas paredes e expostas na forma de bonecos de tamanho real no interior do estabelecimento - demonstrando, assim, as várias formas e os vários espaços que são utilizados pela indústria farmacêutica para a 
divulgação maciça de seus produtos. Também se observou a disposição estratégica de alguns produtos, de forma a fazer com que o usuário percorra o maior caminho possível dentro do estabelecimento a fim de que as compras por impulso sejam aumentadas. Ainda foi possível perceber a exposição de grandes marcas para chamar a atenção do usuário. Essas observações estão de acordo com o que aponta $\mathrm{Memó}$ ria $^{26}$ ao analisar as técnicas oriundas do merchandising e sua relação com o aumento do consumo.

O percentual de peças que descumpre as exigências da legislação vigente e os tipos de infração observados estão de acordo com a literatura. Adibe et al. ${ }^{27}$, analisando panfletos e material promocional coletado em farmácias e drogarias na Nigéria, obtiveram resultados semelhantes. O mesmo foi observado por pesquisadores brasileiros ao analisar a propaganda veiculada em diferentes mídias ${ }^{28},{ }^{29}$. Esses resultados sugerem a importância do fortalecimento da ação de fiscalização das propagandas, a fim de que as propostas da legislação vigente sejam efetivas na proteção do consumidor. Esta ponderação é reforçada pelos resultados obtidos pelo projeto de monitoração, em que se observou, ao longo de suas fases sucessivas, que o número e a frequência das irregularidades foram diminuindo. Isso sugere que uma fiscalização efetiva da qualidade das propagandas poderia contribuir para uma maior adesão às normas sanitárias vigentes, diminuindo o risco a que os usuários são expostos diariamente, uma vez que a propaganda de medicamentos é umas das principais fontes de informação por parte da população ${ }^{30}$.

Outro aspecto importante é apontado por Nascimento ${ }^{31}$ quando pondera que a análise a posteriori da adequação das peças publicitárias, ou seja, apenas após a exposição do público ao conteúdo veiculado na peça publicitária, favorece a exposição da população aos impactos da informação enviesada. $\mathrm{O}$ autor sopesa, ainda, que em países como Espanha e França, há a necessidade de uma autorização a priori das peças, seja qual for o meio de comunicação. Já na Suíça, a autorização prévia é necessária para propagandas veiculadas na TV e no rádio.

\footnotetext{
${ }^{26}$ MEMÓRIA, Paula Roberta Fernandes. 0 merchandising em ponto-de-venda como ferramenta de estratégia para vendas no varejo. Revista Eletrônica da Faculdade Metodista Granbery, n. 8, jan./jun. 2010. Disponível em: <http://re.granbery.edu.br/artigos/MzY1>.

${ }^{27}$ ADIBE, Maxwell Ogochukwu; IGBOELI, NnekaUchenna; UBAKA, Chukwuemeka Michael; UDEOGARANYA, Patrick Obinna; ONWUDIWE, NkejahPrecious; ITA, OboOkokon. op. cit.

${ }^{28}$ BATISTA, Almária Mariz; CARVALHO, Maria Cleide Ribeiro Dantas. Avaliação da propaganda de medicamentos veiculada em emissoras de rádio. Ciência \& Saúde Coletiva, v. 18, n. 2, p. 553-561, 2013. Disponível em: <http://www.scielo.br/pdf/csc/v18n2/27.pdf>. http://dx.doi.org/10.1590/S141381232013000200027.

${ }^{29}$ SILVA, Juliana Fróes da Cruz. Monitoramento da propaganda de medicamentos direcionada ao público leigo, veiculada em meio televiso, frente a RDC 96/2008. 2015. Dissertação (Mestrado) - Faculdade de Farmácia, Universidade Federal Fluminense. Niterói, 2015.

${ }^{30}$ SATO, Miriam Jeiko de S. A propaganda e a publicidade de medicamentos e a informação ao consumidor. Revista de Direito Sanitário, Brasil, v. 3, n. 3, p. 89-115, 2002. Disponível em: <https://www.revistas.usp. br/rdisan/article/view/83839/86720>. http://dx.doi.org/10.11606/issn.2316-9044.v3i3p89-115.

${ }^{31}$ NASCIMENTO, Álvaro César, op. cit.
} 
As irregularidades observadas representam risco à saúde dos usuários, uma vez que estimulam a utilização dos medicamentos: (a) incorretos para seu específico estado de saúde; (b) sem necessidade aparente ou de forma "profilaticamente desnecessária"; e (c) com o intuito de alcançarem um padrão de beleza e/ou inteligência embutido nas celebridades presentes nas peças, como ponderam $L u z$ e Coruja ${ }^{32}$.

A observação de duas peças publicitárias sem qualquer infração, embora seja um número reduzido no universo de 379 peças captadas, comprova a viabilidade de cumprimento da legislação, sem detrimento da função de promoção da peça publicitária. Vale ressaltar que essas peças continham todas as informações necessárias ao uso racional dos produtos pelos usuários, em tamanho e visibilidade compatíveis com as minimamente exigidas pela RDC n. 96/2008.

A divulgação de um total de 180 marcas entre as 379 peças do estudo aponta para a centralidade da marca na propaganda e publicidade de medicamentos, em detrimento da divulgação do princípio ativo. A indústria farmacêutica tem como característica a valorização da marca a fim de que, dessa forma, seja criado um vínculo de confiança entre usuário e marca. O estabelecimento dessa relação de confiança tende a facilitar a aceitabilidade de novas tecnologias, tal qual a aquisição de determinados medicamentos como primeira escolha ${ }^{33}$.

O perfil dos grupos terapêuticos identificado está de acordo com o observado por Vitor et al. ${ }^{34}$ e Silva ${ }^{26}$, mostrando que, mesmo em mídias diferentes, há um padrão de quais medicamentos são propagandeados para o grande público. Vale ressaltar que as classes mais citadas nas propagandas em farmácias e drogarias também estão relacionadas a várias interações medicamentosas e contraindicações, o que reforça a necessidade de cuidado na informação veiculada, sobretudo com a minimização dos riscos envolvidos em seu consumo.

Dados do registro de informações de intoxicações publicado pelo Sistema Nacional de Informações Tóxico-Farmacológicas (Sinitox), da Fiocruz, mostram que os medicamentos ocupam o primeiro lugar entre os principais agentes que causam intoxicações humanas no país, com 11.985 casos registrados em 2013, sendo 241 devido à automedicação ${ }^{35}$. Do total de casos registrados no país, aproximadamente

\footnotetext{
${ }^{32}$ LUZ, Annelena Silva; CORUJA, Paula. Um olhar oximorônico da publicidade da "real beleza". Razón y Palabra, Ecuador, n. 93, p. 320-332, abr./jun. 2016. Disponível em: <http://www.revistarazonypalabra. org/index.php/ryp/article/download/20/pdf>.

${ }^{33}$ TERRES, Melina da Silva et al. O papel da confiança na marca na intenção de adoção de novas tecnologias. Revista de Administração e Inovação, São Paulo, v. 7, n. 4, p. 162-185, 2010. Disponível em: <https:// www.revistas.usp.br/rai/article/view/79196/83268>.

${ }^{34}$ VITOR, Ricardo Sozo et al. Padrão de consumo de medicamentos sem prescrição médica na cidade de Porto Alegre, RS. Ciência \& Saúde Coletiva, Rio de Janeiro, v. 13, p. 737-743, 2008. Disponível em: <http://www. scielo.br/pdf/csc/v13s0/a24v13s0.pdf>. http://dx.doi.org/10.1590/S1413-81232008000700024.

${ }^{35}$ SISTEMA NACIONAL DE INFORMAÇÕES TÓXICO-FARMACOLÓGICAS - SINITOX. Fundação Oswaldo Cruz. Casos registrados de intoxicação humana. 2013. Disponível em: <http://www.fiocruz.br/sinitox_novo/ media/b3.pdf> e <http://www.fiocruz.br/sinitox_novo/media/b10.pdf>.
} 
9,5\% estão relacionados à automedicação, a erro de administração, ao uso indevido ou prescrição inadequada ${ }^{31}$. Essa é mais uma razão para a busca da fiscalização adequada das propagandas, assegurando o equilíbrio entre o estímulo do consumo por meio de exageros aos benefícios dos medicamentos e a cautela em sua utilização, propiciada pela divulgação adequada de cuidados e contraindicações.

O ponto da legislação mais vezes infringido foi o artigo 22, inciso VI, que versa sobre a data de impressão das peças publicitárias. Silva ${ }^{26}$ verificou que essa norma também não foi respeitada na mídia televisiva, sendo desrespeitada em 97\% das vezes. Este dado está em acordo com o que foi observado por Adibe et. al. ${ }^{36}$, analisando propagandas veiculadas em farmácias da Nigéria. A ausência da data de impressão dificulta a ação do órgão sanitário, uma vez que pode favorecer a alegação de que a peça (analisada a posteriori no caso do Brasil) seja antiga ou já devesse estar fora de circulação; assim, dificulta a identificação da legislação em vigor no momento da circulação da peça e a respectiva aplicação de sanções cabíveis.

A qualidade visual das peças também foi questionada por Adibe et al. ${ }^{37}$, concordando com os resultados observados neste estudo, os quais evidenciaram que informações que podem reduzir os impulsos de consumo (como as contraindicações, por exemplo) se encontram, geralmente, pouco visíveis.

Entre os pontos da legislação menos infringidos pelas peças publicitárias analisadas (Tabela 2), estava a inclusão de imagens de pessoas fazendo uso dos medicamentos (art. $8^{\circ}$, III), com apenas duas peças infringindo tal inciso. Uma possível razão para esse resultado está pautada na facilidade de identificação da irregularidade, tanto por parte da fiscalização quanto da população em geral. Duas peças analisadas se utilizavam de expressões ou imagens que pudessem sugerir que a saúde de uma pessoa pode ser afetada pelo não uso do medicamento (art. $\left.8^{\circ}, \mathrm{XI}\right)$. Essa também pode ser considerada uma infração de fácil visualização, uma vez que dificilmente uma propaganda com esse tipo de irregularidade passa despercebida aos olhos da fiscalização e da população em geral. Uma única peça sugeria que o medicamento era a única forma de tratamento disponível e/ou que hábitos de vida saudáveis e/ou a consulta ao médico são supérfluos (o que é proibido de acordo com o art. 26, II). O motivo pelo qual esse artigo pode ter sido pouco infringido se sustenta no fato de que atividades esportivas e uma alimentação balanceada são práticas já difundidas como saudáveis para a maior parte da população e que, juntas, tendem a trazer uma série de benefícios em curto, médio e longo prazo $^{38}$. Sendo assim, uma propaganda que venha a tentar desmistificar esse fato corre o risco de

\footnotetext{
${ }^{36}$ ADIBE, Maxwell Ogochukwu; IGBOELI, NnekaUchenna; UBAKA, Chukwuemeka Michael; UDEOGARANYA, Patrick Obinna; ONWUDIWE, NkejahPrecious; ITA, OboOkokon. op. cit.

${ }^{37}$ Id. Ibid.

${ }^{38}$ DREWNOWSKI, Adam; EVANS, Willian. Nutrition, physical activity, and quality of life in older adults: summary. Journals of Gerontology, EUA, v. 56, n. 2, p. 89-94, Oct. 2001. Disponível em: <https://academic.oup.com/ biomedgerontology/article/56/suppl_2/89/581109>.https://doi.org/10.1093/gerona/56.suppl_2.89.
} 
ter sua credibilidade abalada por veicular informações que vão de encontro ao que está cientificamente comprovado.

Embora haja um intenso debate sobre o direito do consumidor à informação e sobre o papel da propaganda em seu empoderamento para a escolha de seu tratamento ou mesmo para sua adesão ao tratamento ${ }^{39},{ }^{40}$, a baixa qualidade do material promocional observado coloca em cheque esse argumento, uma vez que expõe o usuário ao risco de decisões errôneas e ao uso irracional de medicamentos.

\section{Conclusão}

A metodologia empregada permitiu atender ao objetivo proposto pelo trabalho, identificando problemas importantes na qualidade da propaganda de medicamentos veiculada em farmácias e drogarias. Vários dos pontos da legislação mais vezes infringidos se referem a informações necessárias ao uso racional de medicamentos, tais como advertências obrigatórias, indicações e até mesmo a legibilidade destas e outras informações importantes, podendo, assim, corroborar o mau uso de tais tecnologias.

O cenário observado, embora as peças tenham sido coletadas em uma única cidade e por um período de tempo relativamente curto (principais limitações deste trabalho), sugere a necessidade de aprimoramento do processo de fiscalização sanitária para que se tenha um balanceamento entre o estímulo ao consumo e a promoção do uso racional de medicamentos.

\section{Referências}

ABDALLA, Marcela Campos Esqueff. Monitoramento da propaganda de medicamentos direcionada a profissionais de saúde do Município de Niterói-RJ. 2015. Dissertação (Mestrado) - Faculdade de Farmácia, Universidade Federal Fluminense, Niterói, 2015.

ADIBE, Maxwell Ogochukwu; IGBOELI, NnekaUchenna; UBAKA, Chukwuemeka Michael; UDEOGARANYA, Patrick Obinna; ONWUDIWE, NkejahPrecious; ITA, OboOkokon. Evaluation of information contained in drug advertisement and promotion materials in Nigeria. Tropical Journal of Pharmaceutical Research, v. 14, n. 3, p. 539-544, 2015. Disponível em: <https://www.ajol.info/index. php/tjpr/article/download/115797/105359>. http://dx.doi.org/10.4314/tjpr.v14i3.25.

AL-AGEEL, Sinaa Abdul Mohsen; AL-SABHAN, Jawza Fahad, SULTAN, NohaYahia. Analysis of written advertising material distributed through community pharmacies in Riyadh, Saudi Arabia. PharmPract, Granada, v.11, n. 3, p. 138-143, Jul./Sep. 2013. Disponível em: <https:// www.ncbi.nlm.nih.gov/pmc/articles/PMC3809137/pdf/pharmpract-11-138.pdf $>$.

\footnotetext{
${ }^{39}$ PECHMANN, Cornelia; CATLIN, Jesse R. op. cit.

${ }^{40}$ O'DONOGHUE, Amie C. , SULLIVAN, Helen W., AIKIN, Kathryn J., CHOWDHURY, Dhuly, MOULTRIE, Rebecca R, RUPERT, Douglas J. Presenting efficacy information in direct-to-consumer prescription drug advertisements. Patient Education and Counseling, v. 95, p. 271-280, 2014
} 
AZEVEDO, Germana Santos; PEREIRA, Orcione Aparecida Vieira. Análise da publicidade das propagandas de medicamentos veiculadas em emissoras de televisão. Farmácia \& Ciência, Brasil, v. 1, p. 3-15, 2010. Disponível em: <https://www.unilestemg.br/farmaciaeciencia/ volumes/artigo_1_F_C.pdf>.

BATISTA, Almária Mariz; CARVALHO, Maria Cleide Ribeiro Dantas. Avaliação da propaganda de medicamentos veiculada em emissoras de rádio. Ciência \& Saúde Coletiva, v. 18, n. 2, p. 553-561, 2013. Disponível em: <http://www.scielo.br/pdf/csc/v18n2/27.pdf >. http://dx.doi.org/10.1590/S1413-81232013000200027.

BRASIL. Ministério da Saúde. Agência Nacional de Vigilância Sanitária - ANVISA. Monitoração de propaganda: relatório final do projeto de monitoração. Brasília, 2010. Disponível em: <http://portal.anvisa.gov.br/documents/33864/285104/RelatorioGestaoGPROP_2010_ baixa_11072011.pdf/1972dc29-b15f-48af-854b-4403f8ae697d?version=1.0>.

Ministério da Saúde. Agência Nacional de Vigilância Sanitária - ANVISA. Gerência de Monitoramento e Fiscalização de Propaganda, Publicidade, Promoção e Informação de Produtos Sujeitos à Vigilância Sanitária. Manual: monitoramento de propaganda de produtos sujeitos à vigilância sanitária. Brasília-DF: Ministério da Saúde, 2005. (Série A. Normas e Manuais Técnicos). Disponível em: <http://www.anvisa.gov.br/propaganda/manual_propaganda.pdf >.

BULÁRIO ELETRÔNICO ANVISA. Disponível em: <http://www.anvisa.gov.br/datavisa/ fila_bula/index.asp>. Acesso em: 01 jul. 2016.

BULAS MED. Disponível em: <http://www.bulas.med.br/p/bulas-de-medicamentos/>. Acesso em: 01 jul. 2016.

CARVALHO, Marselle Nobre; BARROS, José Augusto Cabral. Propagandas de medicamentos em revistas femininas. Saúde em Debate, Rio de Janeiro, v. 37, n. 96, p. 76-83, jan./mar. 2013. Disponível em: <http://www.scielo.br/pdf/sdeb/v37n96/09.pdf>. http://dx.doi.org/10.1590/S0103-11042013000100009.

DEL MONTE, Michele Helena Lopes. A influência da publicidade televisiva no consumo da população de classe C: os anunciantes do varejo popular. 2011. Dissertação (Mestrado) - Escola de Comunicações e Artes, Universidade de São Paulo. São Paulo, 2011.

DREWNOWSKI, Adam; EVANS, Willian. Nutrition, physical activity, and quality of life in older adults: summary. Journals of Gerontology, EUA, v. 56, n. 2, p. 89-94, Oct. 2001. Disponível em: <https://academic.oup.com/biomedgerontology/article/56/suppl_2/89/581109>.https:// doi.org/10.1093/gerona/56.suppl_2.89.

DRUGDEX. MicromedexDatabase. Disponível em: <http://www.library.auckland.ac.nz/ databases/?FROM_REF=BN>. Acesso em: 18 jul. 2016.

FROSCH, Dominick L, GRANDE, David; TARN, Derjung M.; KRAVITZ, Richard L. A decade of controversy: balancing policy with evidence in the regulation of prescription drug advertising. American Journal of Public Health, v. 100, n. 1, p. 24-32, 2010. Disponível em: <https://www. ncbi.nlm.nih.gov/pmc/articles/PMC2791253/pdf/24.pdf>.10.2105/AJPH.2008.153767. 
GALATO, Dayani; PEREIRA, Greicy Borges; VALGAS, Cleidson. Análise de Informes Publicitários Distribuídos em Farmácias e Drogarias. Revista de Saúde Pública, São Paulo, v. 45, n. 1, p. 212-215, fev. 2011. Disponível em: <http://www.scielo.br/pdf/rsp/v45n1/en_1466. pdf>.http://dx.doi.org/10.1590/S0034-89102011000100024.

GELLAD, Ziad F., LYLES, Kenneth W. Direct-to-consumer advertising of pharmaceuticals. The American Journal of Medicine, v. 120, p. 475-480, Jun. 2007. 10.1016/j. amjmed.2006.09.030.

GEYER, Robert. The politics of EU health policy and the case of direct-to-consumer advertising for prescription drugs. British Journal of Politics and International Relations (BJPIR), v. 13, n. 4, p. 586-602, Nov. 2011.10.1111/j.1467-856X.2011.00460.x.

GOOGLEMAPS.COM Disponível em: <https://www.google.com.br/maps/ preview? source=newuser-ws $>$. Acesso em: 07 out. 2015.

KORENSTEIN, Deborah, KEYHANI, Salomeh, MENDELSON, Ali, ROSS, Joseph S. Adherence of Pharmaceutical Advertisements. Medical Journals to FDA Guidelines and Content for Safe Prescribing. PloS ONE, v. 6, n. 8 e23336, Aug. 2011. Disponível em: <http:// journals.plos.org/plosone/article/file?id=10.1371/journal.pone.0023336\&type=printable $>$. https://doi.org/10.1371/journal.pone.0023336.

LÈFREVE, Fernando. A função simbólica dos medicamentos. Revista de Saúde Pública, São Paulo, v. 17, n. 6, p. 500-503, 1983. Disponível em: <http://www.scielo.br/pdf/rsp/v17n6/07. pdf>. http://dx.doi.org/10.1590/S0034-89101983000600007.

LUZ, Annelena Silva; CORUJA, Paula. Um olhar oximorônico da publicidade da "real beleza". Razón y Palabra, Ecuador, n. 93, p. 320-332, abr./jun. 2016. Disponível em: <http://www. revistarazonypalabra.org/index.php/ryp/article/download/20/pdf $>$.

MEDICINA NET. Disponível em: <http://www.medicinanet.com.br/categorias/bulas_ remedios.htm>. Acesso em: 01 jul. 2016

MEMÓRIA, Paula Roberta Fernandes. O merchandising em ponto-de-venda como ferramenta de estratégia para vendas no varejo. Revista Eletrônica da Faculdade Metodista Granbery, n. 8, p. 1-22, jan./jun. 2010. Disponível em: <http://re.granbery.edu.br/artigos/MzY1>.

NASCIMENTO, Álvaro César. Propaganda de medicamentos no Brasil:épossível regular? Ciência \& Saúde Coletiva, Brasil, v. 14, n. 3, p. 869-877, maio/jun. 2009. Disponível em: <http://www. scielo.br/pdf/csc/v14n3/en_22.pdf>. http://dx.doi.org/10.1590/S1413-81232009000300022.

NEVES, Aline Souza; LYRA, Divaldo Pereira; CERQUEIRA, Karine Santos; MARCELLINE, Paulo Sérgio; MARQUES, Tatiane Cristina; BARROS, José Augusto Cabral. Influência da propaganda na utilização de medicamentos em grupo de idosos atendidos em uma Unidade Básica de Saúde em Aracaju (SE, Brasil). Ciência \& Saúde Coletiva, Brasil, v. 15, n. 3, p. 3497-3505, 2010. Disponível em: <http://www.scielo.br/pdf/csc/v15s3/v15s3a24.pdf>. http://dx.doi.org/10.1590/S1413-81232010000900024. 
O'DONOGHUE, Amie C.; SULLIVAN, Helen W.; AIKIN, Kathryn J.; CHOWDHURY, Dhuly; MOULTRIE, Rebecca R.; RUPERT, Douglas J. Presenting efficacy information in direct-toconsumer prescription drug advertisements. Patient Education and Counseling, v. 95, n. 2, p. 271-280, 2014. 10.1016/j.pec.2013.12.010.

PECHMANN, Cornelia; CATLIN, Jesse R.The effects of advertising and other marketing communications on health-related consumer behaviors. Current Opinion in Psychology USA, v.10, p. 44-49, 2016. https://doi.org/10.1016/j.copsyc.2015.12.008.

RANDOM NUMBER GENARATOR Disponível em: < http://randomnumbergenerator. intemodino.com/pt/gerador-de-numeros-aleatorios.html>. Acesso em: 08 out. 2015.

SATO, Miriam Jeiko de S. A propaganda e a publicidade de medicamentos e a informação ao consumidor. Revista de Direito Sanitário, Brasil, v. 3, n. 3, p. 89-115, 2002. Disponível em: $<$ https://www.revistas.usp.br/rdisan/article/view/83839/86720>. http://dx.doi.org/10.11606/ issn.2316-9044.v3i3p89-115.

SILVA, Juliana Fróes da Cruz. Monitoramento da propaganda de medicamentos direcionada ao público leigo, veiculada em meio televiso, frente a RDC 96/2008. 2015. Dissertação (Mestrado) - Faculdade de Farmácia, Universidade Federal Fluminense. Niterói, 2015.

SINDICATO DA INDÚSTRIA DE PRODUTOS FARMACÊUTICOS (São Paulo). Disponível em: <http://www.sindusfarmacomunica.org.br/indicadores-economicos/>. Acesso em: 05 ago. 2013.

SISTEMA NACIONAL DE INFORMAÇÕES TÓXICO-FARMACOLÓGICAS - SINITOX. Fundação Oswaldo Cruz. Casos registrados de intoxicação humana. 2013. Disponível em: $<$ http://www.fiocruz.br/sinitox_novo/media/b3.pdf $>$ e $<$ http://www.fiocruz.br/sinitox_novo/ media/b10.pdf $>$.

SUH, Hae Sun; LEE, Donghyun; KIM, Sam Yong; CHEE, Dong Hyun; KANG, Hye-Young. Direct-to-consumer advertising (DTCA) for prescription drugs: Consumers' attitudes and preferences concerning its regulation in South Korea. Health Policy, v. 101, p. 260-268, 2011. 10.1016/j.healthpol.2011.05.005.

TELELISTAS.NET. Disponível em: <http://www.telelistas.net/>. Acesso em: 01 out. 2014.

TERRES, Melina da Silva et al. O papel da confiança na marca na intenção de adoção de novas tecnologias. Revista de Administração e Inovação, São Paulo, v. 7, n. 4, p. 162-185, 2010. Disponível em: <https://www.revistas.usp.br/rai/article/view/79196/83268>.

VITOR, Ricardo Sozo et al. Padrão de consumo de medicamentos sem prescrição médica na cidade de Porto Alegre, RS. Ciência \& Saúde Coletiva, Rio de Janeiro, v. 13, p. 737-743, 2008. Disponível em: <http://www.scielo.br/pdf/csc/v13s0/a24v13s0.pdf>. http://dx.doi.org/10.1590/S1413-81232008000700024. 
Propaganda de medicamentos isentos de prescrição em farmácias comunitárias

Patricky Santos Silva - Mestrando em Ciências Aplicadas a Produtos para a Saúde pela Universidade Federal Fluminense (UFF); especialista em Gestão Pública da Saúde pela UFF. Farmacêutico. Niterói/RJ, Brasil.

Brena da Costa Campos Rangel - Graduanda em Farmácia pela Universidade Federal Fluminense. Pesquisadora do Centro de Apoio à Terapia Racional pela Informação Medicamentos da UFF. Niterói/RJ, Brasil.E-mail: brenacampos@id.uff.br.

Selma Rodrigues de Castilho - Doutora e mestre em Engenharia Biomédica pela Universidade Federal do Rio de Janeiro; graduada em Farmácia pela Universidade Federal Fluminense (UFF). Professora titular da Faculdade de Farmácia da UFF. Niterói/RJ, Brasil. E-mail: selmarc@id.uff.br. 